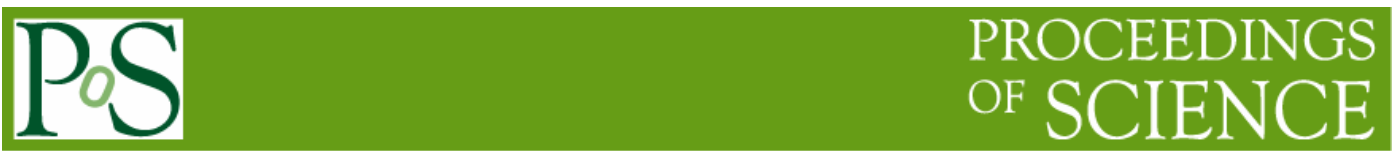

\title{
AMANDE accelerator energy performances
}

\author{
V. Gressier ${ }^{*}$, B. Asselineau, J.F. Guerre-Chaley, A. Martin, H. Muller \\ Institut de Radioprotection et de Sûreté Nucléaire \\ BP3, 13115 St-Paul-Lez-Durance, France \\ E-mail: vincent.gressierlirsn.fr, bruno.asselineaulirsn.fr, jean- \\ francois.guerre-chaleydirsn.fr, alain.martindirsn.fr, \\ hubert.mullerdirsn.fr.
}

The aim of the AMANDE facility of the Institute for Radiological Protection and Nuclear Safety is the production of standard mono-energetic neutron fields between $2 \mathrm{keV}$ and $20 \mathrm{MeV}$ using nuclear reactions between proton or deuteron beams and thin target of selected materials. The neutron field energy is strongly dependent of the charged particles beam energy and energy spread. Therefore, the ion beam characteristics have to be precisely determined. The neutron field reference energy will be established by the time of flight method using a pulsed beam. In that case, the pulse width of the beam influences directly the neutron energy resolution.

The ion beam energy calibration has been performed through the measurements of the magnet factors linking the beam energy and the magnetic field in a $90^{\circ}$ analysing magnet. The pulse width has been measured with a fast Faraday cup and the photon emission measurement at the target with a $\mathrm{BaF} 2$ counter and fast timing electronics. The measured energy performances of the AMANDE charged particles beam offer the possibility to generate neutron fields with only minor uncertainties issued from the accelerator beam energy. In addition, the sub-nanosecond pulse width allows high resolution time of flight measurements.

International Workshop on Fast Neutron Detectors

University of Cape Town, South Africa

April 3-6, 2006

\footnotetext{
${ }^{*}$ Speaker 


\section{Introduction}

To calibrate or investigate neutron measuring devices, the response of these instruments with the neutron energy has to be known. The best method to determine this response is to use monoenergetic neutrons. These monoenergetic neutron fields within the neutron energy range from about $2 \mathrm{keV}$ up to $20 \mathrm{MeV}$ are produced at the AMANDE facility [1], [2].

The AMANDE facility is based on a HVEE 2 MV Tandetron accelerator system delivering proton or deuteron beams, in the energy range $100 \mathrm{keV}-4 \mathrm{MeV}$, in a $\mathrm{DC}$ or in a pulsed mode [3]. This latter mode is used for time of flight experiments in open geometry.

The neutrons are produced by nuclear interaction of accelerated protons or deuterons on thin targets of scandium, lithium (or lithium fluorite), deuterium or tritium in titanium. The targets are placed at the end of the beamline on a wobbling target holder with an air cooling system.

Neutrons are producing in the whole solid angle. However, for a given proton or deuteron beam energy, a unique neutron energy is observed at a defined angle. There is a full correlation between proton or deuteron energy and the emitted neutron energy. To define precisely the monoenergetic neutron field mean energy and width, the ion beam energy and energy spread have to be precisely characterized.

The neutron field reference energy calculated from nuclear kinematics has to be experimentally validated by neutron time of flight measurements to be traceable to primary standards. Indeed, uncertainties on target thickness, chemical composition and purity can induce noticeable systematic deviations. In time of flight experiments, the energy resolution depends mainly on two parameters: detector thickness and pulse width. This latter parameter has to be as small as possible and precisely determined.

This study is therefore focused, in AMANDE DC mode, on the ion beam energy calibration and the ion beam energy spread value, as well as the determination of the achievable beam pulse width in pulsed mode.

\section{Ion beam energy calibration}

\subsection{Method for the determination of the ion beam energy}

Into a beam, the charged particles have a Gaussian-like energy distribution which can be defined by its mean energy and its width. The mean energy is considered as the energy of the beam whereas the width of the Gaussian distribution is the energy spread of the beam.

The beam width is mainly due to the fluctuation of the accelerator high voltage (voltage ripple) and to the energy broadening effect due the ion beam interactions within the stripper canal of the accelerator [4].

The mean energy of the beam is not defined by the high voltage applied to the accelerator as this method is not sufficiently accurate and stable with time, but by a 90-degree magnet using the well known direct relation between beam energy $E$ (in $\mathrm{MeV}$ ) and magnetic field $B$ into the $90^{\circ}$ magnet (in T):

$$
E=\frac{R}{2 m} B^{2}=k \cdot B^{2}
$$


$R$ is the radius of the circular trajectory of the particles into the magnet and $m$ the ion mass. $k$ is the magnet factor and is the parameter which will be experimentally determined in this study.

To define the trajectory of the beam (i.e. to fix $R$ ), beam position stabilizing slits systems in the x-plane are incorporated at two places. The first slits system is implemented before the $90^{\circ}$ magnet to have the beam enter of the $90^{\circ}$ magnet at a fixed position by controlling the $\mathrm{X}$ steerer situated just after the accelerator. The second one is positioned directly behind the $90^{\circ}$ degree magnet. This latter one control the high voltage applied to the accelerator terminal. To define more precisely the trajectory, two apertures are placed into the beamline when tuning the beam.

To determine precisely the magnetic field, a computer controlled NMR teslameter is used with three gauges placed at the center of the magnet cavity allowing to cover the magnetic field range from $90 \mathrm{mT}$ to $965 \mathrm{mT}$.

To determine in an absolute way the relation between energy and magnetic field nuclear reactions are used. The beam energy calibration is performed using $\gamma$-ray resonances and neutron threshold reactions listed in table 1.

Table 1: Summary of selected calibration points and corresponding targets In case of $\boldsymbol{\gamma}$-ray resonances, the natural width is given.

\begin{tabular}{|c|c|c|c|c|}
\hline Reaction & $\begin{array}{l}\text { Ion energy } \\
(\mathrm{keV})\end{array}$ & $\begin{array}{l}\text { Resonance natural width } \\
(\mathrm{keV})\end{array}$ & Reference & $\begin{array}{c}\text { Target / } \\
\text { Thickness }\left(\mu \mathrm{g} \cdot \mathrm{cm}^{-2}\right)\end{array}$ \\
\hline${ }^{19} \mathrm{~F}(\mathrm{p}, \alpha \gamma){ }^{16} \mathrm{O}$ & $340.46 \pm 0.04$ & $2.34 \pm 0.04$ & [5] & ${ }^{7} \mathrm{LiF} / 45.3$ \\
\hline${ }^{27} \mathrm{Al}(\mathrm{p}, \gamma){ }^{28} \mathrm{Si}$ & $632.23 \pm 0.04$ & $0.0048 \pm 0.0004$ & [6] / [7] & ${ }^{27} \mathrm{Al} / 113.9$ \\
\hline${ }^{27} \mathrm{Al}(\mathrm{p}, \gamma){ }^{28} \mathrm{Si}$ & $991.756 \pm 0.017$ & $0.100 \pm 0.015$ & {$[8] /[7]$} & ${ }^{27} \mathrm{Al} / 113.9$ \\
\hline${ }^{3} \mathrm{H}(\mathrm{p}, \mathrm{n}){ }^{3} \mathrm{He}$ & $1018.8751 \pm 0.0015$ & - & [9] & $\mathrm{TiT} / 215$ \\
\hline${ }^{27} \mathrm{Al}(\mathrm{p}, \gamma){ }^{28} \mathrm{Si}$ & $1316.87 \pm 0.03$ & $0.070 \pm 0.035$ & [8] / [7] & ${ }^{27} \mathrm{Al} / 113.9$ \\
\hline${ }^{27} \mathrm{Al}(\mathrm{p}, \gamma){ }^{28} \mathrm{Si}$ & $1587.49 \pm 0.08$ & $<0.200$ & [6] & ${ }^{27} \mathrm{Al} / 113.9$ \\
\hline${ }^{13} \mathrm{C}(\mathrm{p}, \gamma){ }^{14} \mathrm{~N}$ & $1746.8 \pm 0.3$ & $0.077 \pm 0.012$ & {$[10] /[11]$} & ${ }^{13} \mathrm{C} / 95$ \\
\hline${ }^{27} \mathrm{Al}(\mathrm{p}, \gamma){ }^{28} \mathrm{Si}$ & $1799.75 \pm 0.09$ & $<0.200$ & [6] & ${ }^{27} \mathrm{Al} / 113.9$ \\
\hline${ }^{7} \mathrm{Li}(\mathrm{p}, \mathrm{n})^{7} \mathrm{Be}$ & $1880.36 \pm 0.08$ & - & [9] & ${ }^{7} \mathrm{LiF} / 45.3$ \\
\hline${ }^{13} \mathrm{C}(\mathrm{p}, \mathrm{n}){ }^{13} \mathrm{~N}$ & $3235.48 \pm 0.29$ & - & [9] & ${ }^{13} \mathrm{C} / 95$ \\
\hline
\end{tabular}

\subsection{Experimental set-up}

Photon energy distributions from the $\gamma$-ray resonances are measured with a BGO detector [12]. For neutron threshold measurements, a 4.2"' Bonner sphere is used with a $0.5 \mathrm{NH} 1 / 1 \mathrm{KI}$ ${ }^{3} \mathrm{He}$ counter [13], as the response of this detector does not vary by more than $2.2 \%$ in the neutron energy range of interest ${ }^{*}$. Placed at $8.5 \mathrm{~cm}$ from the target, the sphere sustains a sufficient half angle at the target to measure all emitted neutrons over a few $\mathrm{keV}$ ion energy range above threshold.

Variation of the beam energy has been performed by applying a variable voltage at the target using a $20 \mathrm{kV}$ high voltage power supply connected directly to the insulated target tube. This voltage is applied through a $20 \mathrm{kV}$ high voltage power supply connected directly to the

\footnotetext{
* $4 \mathrm{keV}$ to $94 \mathrm{keV}$ corresponding to a $5 \mathrm{keV}$ energy range of study above threshold energies.
} 
insulated target tube. This high voltage is controlled in the acquisition room by a source meter and measured on the return signal by a calibrated voltmeter to take into account the voltage generated by the beam current on the target.

Several measuring campaigns were performed between November 2005 and April 2006 to study the long term energy reproducibility in order to deduce the beam energy uncertainty.

\subsection{Data analysis}

The counts $\mathrm{Y}$ in each detector as a function of the high voltage HV applied to the target have been fitted with Origin 7.5 software, weighting each data point by its statistical uncertainty. Examples are given in the figure 1.

The energy dependence of the total cross section for neutron emission just above threshold varies, in the case of neutrons and light nuclei, as $\left(E-E_{0}\right)^{3 / 2}[11]$, where $E_{0}$ is the threshold energy. The experimental data have therefore been fitted using a $\mathrm{Y}^{3 / 2}$ function as well as by a linear fit of the counts elevated to the power $2 / 3$. Mean value of both method with uncertainty taking into account the two fitting ways is considered at the end. These fits have been performed by removing the data points just around the threshold (influence of the beam energy spread).

Ion beam and resonance have Gaussian and Lorentz energy distribution respectively. To simplify the problem, it is assume that the experimental data are described by the cumulative Gaussian or Lorentz distribution if the resonance width is respectively smaller or larger by more than a factor 3 compare to the beam width.
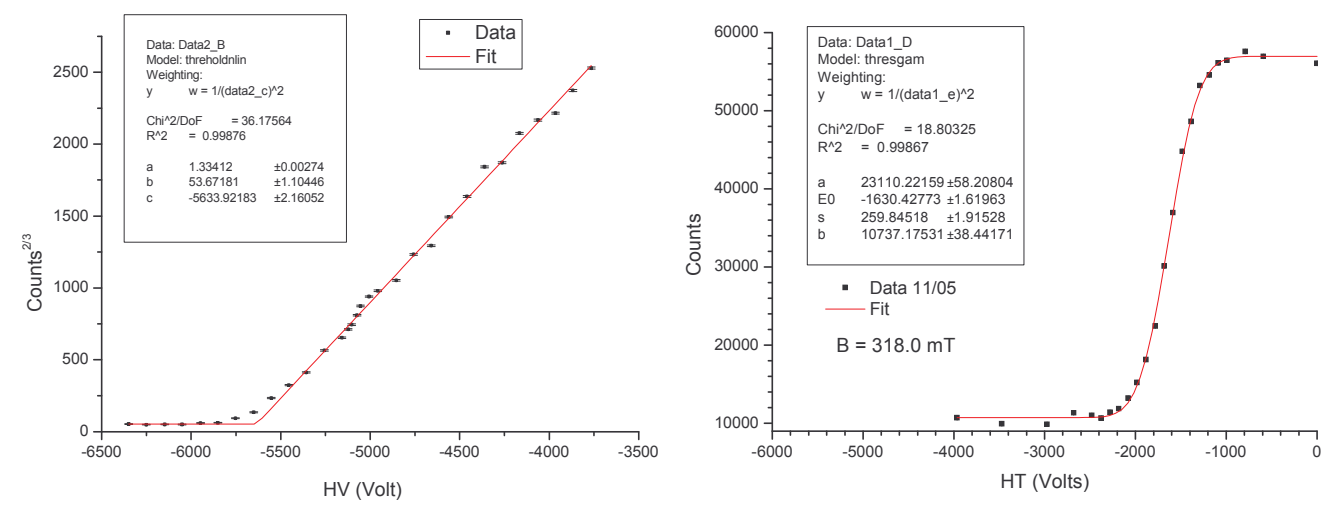

Figure 1: Fits of the experimental data at the ${ }^{7} \mathrm{Li}(\mathrm{p}, \mathrm{n})^{7} \mathrm{Be}$ threshold (left) and the ${ }^{13} \mathrm{C}(\mathrm{p}, \gamma){ }^{14} \mathrm{~N}$ resonance at $1747 \mathrm{keV}$ (right)

From the data analysis, it appears that the magnet factor $k_{p}$, for proton beam, decreases linearly with the proton beam energy. It has been observed that the k-value of magnets systematically increase or decrease with beam energy [10]. AMANDE $90^{\circ}$ magnet is then of the second category. The $k_{p}$ value is determined by fitting the whole set of experimental data (at the exception of the 1587 and $1800 \mathrm{keV}$ resonances) by a linear function plotted in the figure 2 . 


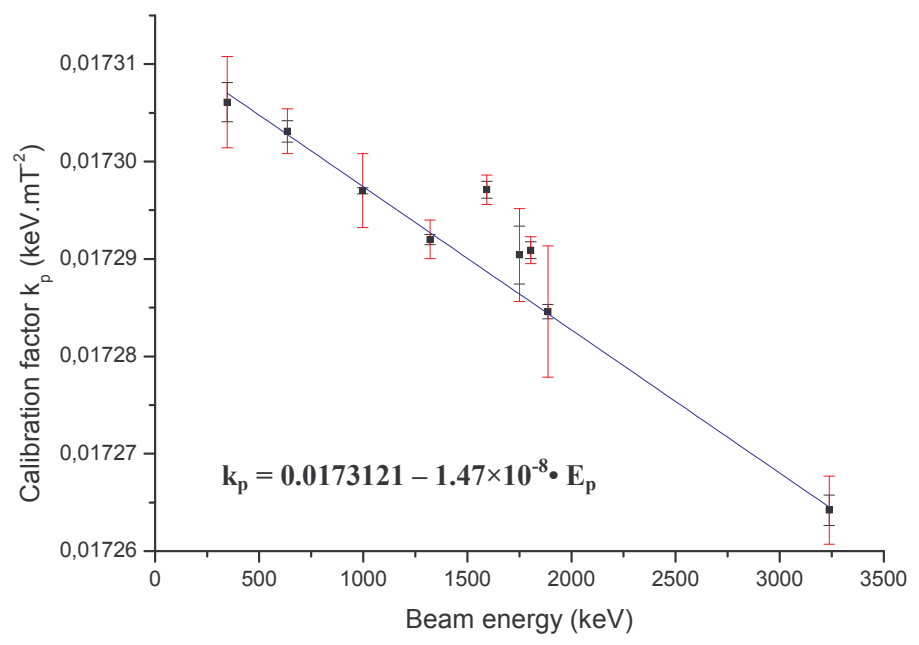

Figure 2: Variation of the magnet factor $k_{p}$ as a function of the proton beam energy. Black uncertainties are derived from the resonance energy uncertainties, whereas red uncertainties are the quadratic sum of the previous uncertainties with the standard deviations of the $k_{p}$ distribution at each energy.

The $k_{p}$ uncertainties are calculated directly from the experimental variance. Due to the small amount of measurements, this uncertainty is multiplied by the appropriate Student coefficient and then quadratically added to the uncertainty coming from the resonance or threshold energies. All theses uncertainties are presented in table 2, as well as the relative deviation between the mean experimental value of the magnet factor and the theoretical value $\mathrm{k}_{\mathrm{p}}{ }^{\text {fit }}$ deduced from the linear fit.

Table 2: Magnet factor and beam energy uncertainties

\begin{tabular}{|c|c|c|c|c|c|c|}
\hline $\begin{array}{c}\text { Threshold } \\
\text { or resonance } \\
\text { energy } \\
(\mathrm{keV})\end{array}$ & $\begin{array}{c}k_{p} \\
\left(\times 10^{-2}\right)\end{array}$ & $\begin{array}{c}\Delta \mathrm{k}_{\mathrm{p}} \\
\text { reproducibility } \\
\left(\times 10^{-6}\right)\end{array}$ & $\begin{array}{c}\Delta \mathrm{k}_{\mathrm{p}} \\
\text { energy } \\
\left(\times 10^{-6}\right)\end{array}$ & $\begin{array}{c}\Delta \mathrm{k}_{\mathrm{p}} \\
\text { total } \\
\left(\times 10^{-6}\right)\end{array}$ & $\begin{array}{l}\Delta \mathrm{k}_{\mathrm{p}} / \mathrm{k}_{\mathrm{p}} \\
\left(\times 10^{-4}\right)\end{array}$ & $\begin{array}{c}\left(\mathrm{k}_{\mathrm{p}}-\mathrm{k}_{\mathrm{p}}{ }^{\mathrm{fit}}\right) / \mathrm{k}_{\mathrm{p}}{ }^{\mathrm{fit}} \\
\left(\times 10^{-4}\right)\end{array}$ \\
\hline 340.46 & 1.73061 & 5.46 & 2.00 & 5.82 & 3.36 & 0.55 \\
\hline 632.23 & 1.73031 & 2.68 & 1.09 & 2.90 & 1.67 & 0.19 \\
\hline 991.756 & 1.72970 & 4.54 & 0.30 & 4.55 & 2.63 & 0.28 \\
\hline 1316.87 & 1.72920 & 2.19 & 0.52 & 2.25 & 1.30 & 0.43 \\
\hline 1587.49 & 1.72971 & 1.62 & 0.87 & 1.84 & 1.07 & 4.87 \\
\hline 1746.8 & 1.72904 & 4.30 & 2.97 & 5.22 & 3.02 & 2.34 \\
\hline 1799.75 & 1.72909 & 1.28 & 0.87 & 1.55 & 0.90 & 3.07 \\
\hline 1880.36 & 1.72846 & 7.67 & 0.74 & 7.70 & 4.46 & 0.12 \\
\hline 3235.48 & 1.72642 & 3.57 & 1.55 & 3.90 & 2.26 & 0.19 \\
\hline
\end{tabular}

The fact that the points have a certain distribution for a given threshold or $\gamma$-ray resonance energy, reveals variations of the beam energy or/and the magnetic field. Possible explanations are the magnetic field differential hysteresis into $90^{\circ}$ magnet and the difference between the field at the NMR probe and the field really seen by the particles through their pathway into the magnet. In this latter case, any variation of beam focusing or alignment at the entrance of the 
magnet will modify the beam trajectory, i.e. its energy due to the control loop with the slits. Further studies are needed about these specific points.

The relative $k_{p}$ uncertainties as well as their deviation from the linear fit of paragraph 7.1 are found lower than $5.0 \times 10^{-4}$ for all energies. The variation of this relative uncertainty with the beam energy and the explanations of the deviation from the linear fit at 1587 and $1800 \mathrm{keV}$ are not straightforward and need further studies.

However, it is possible to consider a constant relative $k_{p}$ uncertainty of:

$$
\Delta k_{p} / k_{p}=\mathbf{5 . 0} \times 10^{-4}=\Delta E_{p} / E_{p}
$$

A $2 \mathrm{MeV}$ ion beam, proton or deuteron, will then have $\pm 1 \mathrm{keV}$ uncertainty which corresponds to the specification of the AMANDE accelerator long term reproducibility as awaited by the manufacturer HVEE.

Further measurement campaigns and future studies about systematic deviations could improve this uncertainty. It is however already well suited for reference monoenergetic neutron fields production above $100 \mathrm{keV}$ as the uncertainty propagated to the neutron energy is lower than $2 \mathrm{keV}$ for the ISO 8529-1 standard neutron energies, as shown in table 3.

Table 3: Beam energy and related neutron energy uncertainty for the ISO 8529-1 standard energies.

\begin{tabular}{cccccc}
\hline Nuclear Reaction & $\begin{array}{c}\mathrm{E}_{\mathrm{n}} \\
(\mathrm{keV})\end{array}$ & $\begin{array}{c}\mathrm{E}_{\text {beam }} \\
(\mathrm{keV})\end{array}$ & $\Delta \mathrm{E}_{\mathrm{n}} / \Delta \mathrm{E}_{\text {beam }}$ & $\begin{array}{c}\Delta \mathrm{E}_{\text {beam }} \\
(\mathrm{keV})\end{array}$ & $\begin{array}{c}\Delta \mathrm{E}_{\mathrm{n}} \\
(\mathrm{keV})\end{array}$ \\
\hline${ }^{45} \mathrm{Sc}(\mathrm{p}, \mathrm{n})$ & $2{ }^{\dagger}$ & 2910 & 1.17 & 1.455 & 1.70 \\
& 24 & 2922 & 1.26 & 1.461 & 1.84 \\
${ }^{7} \mathrm{Li}(\mathrm{p}, \mathrm{n})$ & 144 & 1936 & 1.50 & 0.968 & 1.45 \\
& 250 & 2016 & 1.25 & 1.008 & 1.26 \\
& 565 & 2293 & 1.10 & 1.147 & 1.26 \\
${ }^{3} \mathrm{H}(\mathrm{p}, \mathrm{n})$ & 1200 & 1997 & 1.02 & 0.999 & 1.02 \\
& 2500 & 3281 & 1.01 & 1.641 & 1.66 \\
& 2800 & 3580 & 1.00 & 1.790 & 1.79 \\
${ }^{2} \mathrm{H}(\mathrm{d}, \mathrm{n})$ & 2800 & 78 & 2.52 & 0.039 & 0.10 \\
& 5000 & 1774 & 1.07 & 0.887 & 0.95 \\
${ }^{3} \mathrm{H}(\mathrm{d}, \mathrm{n})$ & 14800 & 111 & 3.77 & 0.056 & 0.21 \\
& 19000 & 2548 & 1.31 & 1.274 & 1.67 \\
\hline
\end{tabular}

For the $2 \mathrm{keV}$ and $24 \mathrm{keV}$ neutron energies, the ion beam uncertainty is too high. One reason is simply a non negligible relative energy uncertainty but the main reason is the fine resonance structure of the ${ }^{45} \mathrm{Sc}(\mathrm{p}, \mathrm{n})$ reaction cross section [14] with the incident proton energy. The $2 \mathrm{keV}$ and $24 \mathrm{keV}$ neutron energies are defined at resonance energies and any variation or deviation of the ion energy can induce strong neutron fluence variations.

However, the usual method at these two particular energies to adjust and control the ion beam energy, is to maintain the neutron fluence rate maximum (using neutron monitors for example), i.e. the proton beam energy at the resonance energy.

In addition, a main uncertainty of the ion beam energy which has not been considered up to now in this study is due to the target itself. Any surfacic contamination or inhomogeneity of

\footnotetext{
${ }^{\dagger}$ This energy is obtained at $60^{\circ}$ with respect to the ion beam direction. At $0^{\circ}$, the neutron energy is of $8 \mathrm{keV}$.
} 
the reactive layer may induce energy shift of several $\mathrm{keV}$ which will change with time, beam power and focus, target storage conditions, etc. To reduce the neutron energy uncertainties below $2 \mathrm{keV}$ will then more need a huge and delicate work about targets than about ion beam energy accurate definition.

\section{Ion beam energy spread}

The beam energy spread has been determined from the fit of the measurements of the $\gamma$-ray resonances with small natural width with the cumulative Gaussian function. This fit leads to the determination of the Gaussian standard deviation. The beam energy spread is obtained by subtracting quadratically the natural resonance width to $2 \times \sigma$, the resonance being considered as a Gaussian in that case.

$\gamma$-ray resonances at $632,992,1317,1587,1747$ and $1800 \mathrm{keV}$ have been used. For the last resonance, a natural width lower than $200 \mathrm{eV}$ is reported in [6]. However, the shape of the measurements is better represented by the cumulative Lorentz function than by the cumulative Gaussian function indicating a larger than expected resonance width: this width has been estimated in this study as about $460 \pm 100 \mathrm{eV}$.

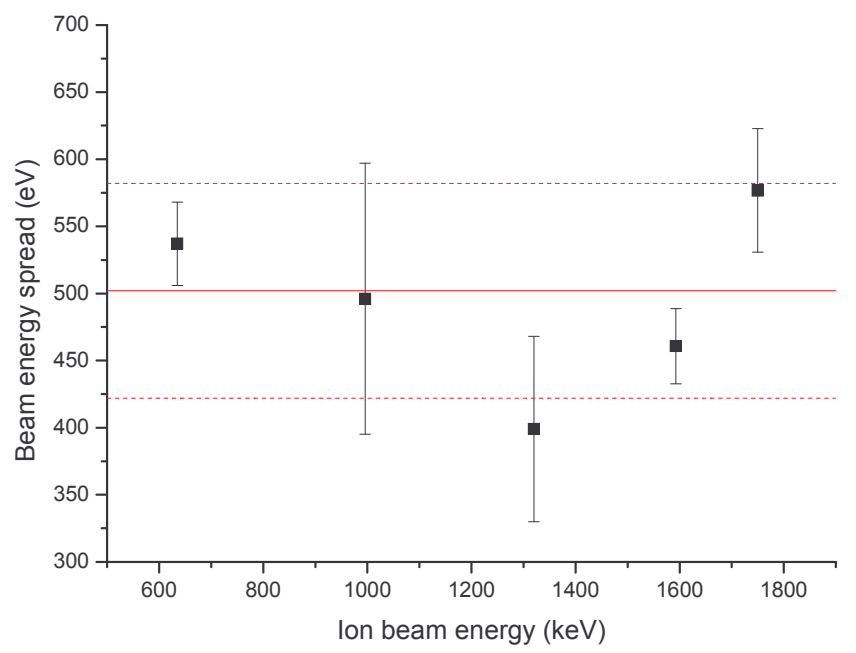

Figure 3: Variation of the proton beam energy spread with energy.

The experimentally determined values of the beam energy spread are shown in the figure 3. This spread appears to be constant in the energy range 632 to $1747 \mathrm{keV}$. The associated uncertainties correspond to the standard deviation of the experimental values obtained for the same resonance during the several measurement campaigns. These uncertainties give an estimation of the variation of the beam energy spread depending of the beam tuning. This variation appears to be lower than $100 \mathrm{eV}$ which is perfectly suitable for AMANDE applications.

The unweighted mean value of all the performed measurements is considered for the final value with the standard deviation as uncertainty. 
The proton beam energy spread $\sigma_{\mathrm{Ep}}$ is then: $\boldsymbol{\sigma}_{\mathrm{Ep}}=\mathbf{5 0 2} \pm \mathbf{8 0} \mathbf{~ e V}$

This value is in good agreement with the awaited $500 \mathrm{eV}$ value specified by the accelerator manufacturer HVEE and is assumed to be valid in the complete energy range of the accelerator $(100 \mathrm{keV}-4 \mathrm{MeV})$ and for both proton and deuteron beams.

\section{Pulse width determination}

Absolute neutron energy references will be determined by time of flight measurements. The possible flight paths are between 0.5 and $6 \mathrm{~m}$ at any angle (and $10 \mathrm{~m}$ at $0^{\circ}$ ). In pulsed mode, the accelerator can run at frequencies between $62.5 \mathrm{kHz}$ and $2 \mathrm{MHz}$.

The pulse width tuning is performed at a fast faraday cup measuring the induced current, and is experimentally determined using a capacitive pick up unit and a fast BaF2 scintillator with a fast timing circuit (based on two $1 \mathrm{GHz}$ amplifiers and timing discriminators Ortec 9327 and TAC Ortec 566 units). By this way, the time separating the detection of photons emitted from the target to the time where the pulse was at the pick-up ring position (about $1 \mathrm{~m}$ before the target) is measured.

Mainly due to space charge effect, the pulse width decreases when the ion beam energy increases. As for neutron monoenergetic fields production, the beam energy is in the $2 \mathrm{MeV}$ energy range, the BaF2 counter has been placed a few $\mathrm{cm}$ in front of a $114 \mu \mathrm{g} / \mathrm{cm}^{2}$ thick aluminium target and a proton beam energy of $2380 \mathrm{keV}$ was used. The time measurements were gated by the BaF2 energy signal in order to select only photons from the $2374 \mathrm{keV}$ ${ }^{27} \mathrm{Al}(\mathrm{p}, \gamma){ }^{28} \mathrm{Si}$ resonance.

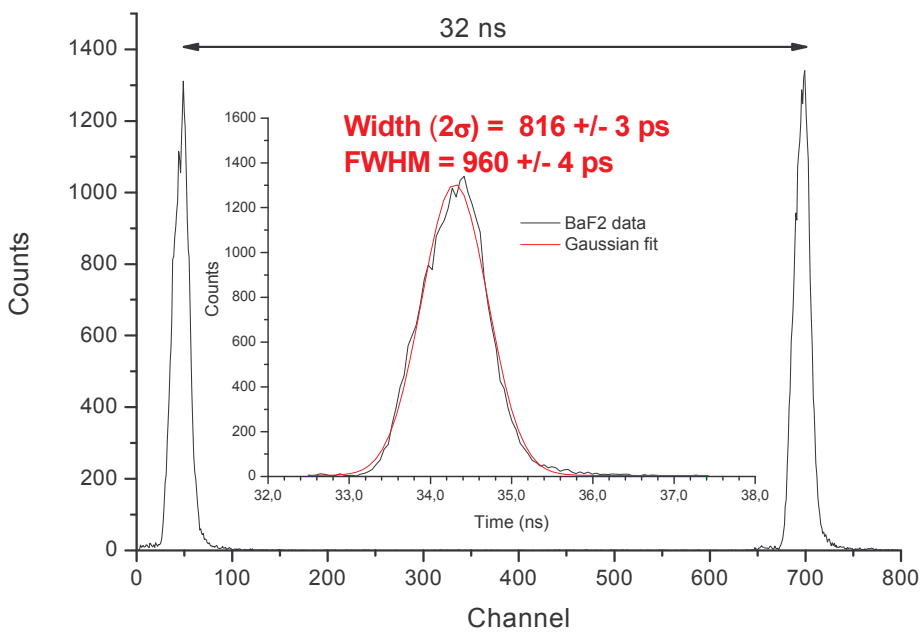

Figure 4: Experimental determination of the pulse width at the $2374 \mathrm{keV}^{27} \mathrm{Al}(\mathrm{p}, \gamma)^{28} \mathrm{Si}$ resonance.

As shown in the figure 4, a pulse width lower than 1 ns has been obtained, allowing neutron time of flight measurement with less than $1 \%$ energy resolution on the whole energy range. The time calibration of the ADC channels have been performed using an Ortec 425A nanosecond delay unit. 


\section{Conclusion}

AMANDE facility is able to deliver ion beams well known in energy for neutron monoenergetic field production. The preliminary relative beam energy uncertainty has been found to be $5 \times 10^{-4}$, leading to a maximum uncertainty of about $2 \mathrm{keV}$ for the ISO standard neutron energies. More details are available in [15]. However, further efforts are needed to reduce systematic effects.

From fast timing measurements, it has been shown that AMANDE can produce pulsed beam with pulse width lower than $1 \mathrm{~ns}$ in the ion beam energy range used to produce most of the standard monoenergetic neutron fields. Precise time of flight measurement with energy resolution between 0.1 and $1 \%$ energy are therefore achievable.

\section{References}

[1] V. Gressier, G. Pelcot, J. L. Pochat and T. Bolognese-Milstajn, New IRSN facilities for neutron production, Nuclear Instruments and Methods in Physics Research A 505 (1-2) (2003) 370.

[2] V. Gressier, J. F. Guerre-Chaley, V. Lacoste, L. Lebreton, G. Pelcot, J. L. Pochat, T. BologneseMilstajn and D. Champion, AMANDE: a new facility for monoenergetic neutron fields production between $2 \mathrm{keV}$ and $20 \mathrm{MeV}$, Radiation Protection Dosimetry 110 (1-4) (2004) 49.

[3] D.J.W Mous, J. Visser and R.G. Haitsma, A nanosecond pulsing system for MeV light ions using a 2 MV Tandetron, Nuclear Instruments and Methods in Physics Research B 219-220, (2004) 490.

[4] B. Hartmann, S. Kalbitzer and Ch. Klatt, Energy spread of ion beams passing a gas stripper, Nuclear Instruments and Methods in Physics Research B 124, (1997) 490.

[5] F. Ajzenberg-Selove, Energy levels of light nuclei $A=18-20$, Nuclear Physics A 475, (1987) 1.

[6] J.W. Maas, E. Somorjai, H.D. Graber, C.A. Van den Wijngaart, C. Van der Leun and P.M. Endt, Investigation of ${ }^{28}$ Si levels with the $(\alpha, \gamma)$ and $(p, \gamma)$ reactions, Nuclear Physics A 301, (1978) 213.

[7] P.M. Endt and C. Van der Leun, Energy levels of $A=21-44$ nuclei (V), Nuclear Physics A 214, (1973) 1.

[8] S.A. Brindhaban, P.H. Barker, M.J. Keeling and W.B. Wood, Accelerator beam energy calibration with the ${ }^{27} \mathrm{Al}(p, n)$ and ${ }^{27} \mathrm{Al}(p, \gamma)$ reactions, Nuclear Instruments and Methods in Physics Research A 340, (1994) 436.

[9] Data produced by the code QCALC, written by T.W. Burrows, National Nuclear Data Center, Brookhaven National Laboratory, and based on the Audi-Wapstra Atomic Mass Tables, A.H. Wapstra, G. Audi, and C. Thibault. Nuclear Physics A729, 129 (2003) and G. Audi, A.H. Wapstra, and C. Thibault. Nuclear Physics A729, 337 (2003). http://www.nndc.bnl.gov/qcalc/

[10] R. Böttger, Energy calibration of the PTB-VdG Accelerator, PTB report 6.41-02-2 (2002).

[11]J.B. Marion, J.L. Fowler, Fast Neutron Physics, Interscience Publishers, London (1960) 1867.

[12]B. Asselineau, BGO as soft gamma-ray detector in mixed field, IRSN Technical note DRPH/SDE/LMDN 2005-01 (2005).

[13] V. Lacoste, V. Gressier, J.-L. Pochat, F. Fernandez, M. Bakali and T. Bouassoule, Characterization of Bonner sphere systems at monoenergetic and thermal neutron fields, Radiation Protection and Dosimetry 110, p 529-532 (2004).

[14] M. Cosack, H. Lesiecki and J.B. Hunt, Monoenergetic neutrons of energies from $0.5 \mathrm{keV}$ to $40 \mathrm{keV}$ via the reaction ${ }^{45} \mathrm{Sc}(p, n){ }^{45} \mathrm{Ti}$, Proceedings of fifth symposium on neutron dosimetry, Munich/Neuherberg 17 -21 September 1984 EUR-9762, Vol I, 597 -616 (1985)

[15] V. Gressier, B. Asselineau, J.-F. Guerre-Chaley, A. Martin and H. Muller, Energy calibration of AMANDE accelerator ion beam, IRSN report DRPH/SDE 2006-14 (2006). 